\title{
Propagación y establecimiento de citronela (Pelargonium graveolens) bajo malla sombra
}

\author{
Propagation and establishment of citronella \\ (Pelargonium graveolens) under shade net \\ Diana Miriam Mc Caughey-Espinoza ${ }^{1}$, Gloria Irma Ayala-Astorga ${ }^{1}$, \\ Génesis V. Buitimea-Cantúa², Nydia E. Buitimea-Cantúa2* y Andrés Ochoa-Meza ${ }^{3}$
}

\section{RESUMEN}

Citronela (Pelargonium graveolens) es una planta aromática medicinal que posee compuestos activos como citronelol y geraniol. El objetivo de este trabajo fue evaluar la propagación de citronela utilizando las hojas y el establecimiento de las nuevas plantas bajo malla sombra. Los tratamientos que se usaron para el enraizamiento fueron suelo y peat moss. Se presentó un mayor enraizamiento en hojas sembradas en suelo (91\%) que en hojas sembradas en peat moss (67\%). Las hojas enraizadas en ambos sustratos mostraron un $91 \%$ de sobrevivencia al trasplante. Sin embargo, las hojas enraizadas en suelo presentaron 10.15 veces mayor longitud de raíces comparado con peat moss, mientras que el número de raíces en las hojas sembradas en suelo y peat moss fue similar. No se observaron diferencias significativas en las mediciones dasométricas (altura, diámetro de copa y diámetro basal) de las plantas de citronela. El rendimiento de materia seca por planta fue mayor 1.07 veces en suelo comparado con peat moss. Durante el enraizamiento y establecimiento de citronela no se registraron plagas ni enfermedades, lo que es benéfico para este cultivo al evitar el uso de agroquímicos. La propagación vegetativa de hojas de citronela fue mejor utilizando el suelo como sustrato, lo que permitió la obtención de nuevas plantas.

Palabras clave: Pelargonium graveolens, citronela, enraizamiento, establecimiento.

\section{ABSTRACT}

Citronella (Pelargonium graveolens) is a medicinal aromatic plant that has bioactive compounds such as citronellol and geraniol. The objective of this work was to evaluate the propagation of citronella using the leaves and the establishment of the new plant under shade net. The treatments that were used for rooting were soil and peat moss. There was a greater rooting in leaves sown in soil (91\%) than in leaves sown in peat moss (67\%). The leaves rooted in both substrates had a $91 \%$ survival after transplantation. However, the leaves rooted in soil presented 10.15 times longer root length compared to peat moss. While the number of roots in the leaves sown in soil and peat moss was similar. No significant changes were observed in the dasometric measurements (height, crown, and basal diameter) of citronella plants. The dry matter yield per citronella plant was 1.07 times higher in soil compared to peat moss. During the rooting and establishment of citronella there were no pests or diseases, which is beneficial for this crop avoiding the use of agrochemicals. The vegetative propagation of citronella leaves was better using the soil as a substrate, which allowed the obtaining of new plants.

Keywords: Pelargonium graveolens, citronella, entanglement, establishment.

\section{Introducción}

A nivel mundial se ha incrementado la demanda del consumo de plantas aromáticas. México es uno de los países del continente americano que presentan un potencial para la producción de plantas aromáticas medicinales. La mayoría de las especies aromáticas y medicinales son producidas para el

\footnotetext{
1 Departamento de Investigaciones Científicas y Tecnológicas de la Universidad de Sonora. Hermosillo Sonora, México.

2 Tecnológico de Monterrey, Centro de Biotecnología-FEMSA, Escuela de Ingeniería y Ciencias, Campus Monterrey. Av. Eugenio Garza Sada 2501, Monterrey, N.L., C.P. 64849, México.

3 Departamento de Agricultura y Ganadería. Universidad de Sonora. Hermosillo Sonora, México.

* Corresponding author: $\underline{\text { nebc@tec.mx }}$
} 
mercado de exportación, y los migrantes mexicanos y asiáticos son los principales consumidores en Estados Unidos y Canadá (Juárez et al., 2013).

Entre los estados productores de plantas aromáticas medicinales que están certificados se encuentran Morelos, Baja California Sur, Baja California, Estado de México, Nayarit, Oaxaca, Puebla y Tlaxcala (Pérez, 2009). Sonora es un estado estratégico para la producción de plantas aromáticas medicinales bajo sombra ya sea en fresco o seco, por ser cultivos rentables y económicamente constituyen una alternativa para el cambio de una agricultura tradicional.

En el ámbito agrícola se buscan día a día otras alternativas de nuevos cultivos, y el de citronela es una opción de explotación. De acuerdo con la Organización Mundial de la Salud (OMS), una gran diversidad de especies vegetales son empleadas principalmente en fitomedicamentos, basándose en estudios etnobotánicos que sustentan el uso tradicional de plantas aromáticas medicinales de acuerdo al sitio de origen. La resistencia que se ha presentado por el uso incorrecto de fármacos antibacterianos ha favorecido la propagación de bacterias capaces de sobrevivir. Por lo tanto, estos medicamentos han ido perdiendo gradualmente su efectividad, lo que ha originado una emergencia sanitaria a nivel mundial (Shankar y Balasubramanium, 2014).

La citronela (Pelargonium graveolens L. Her. ex Ait.) es una especie del género Pelargonium que pertenece a la familia Geraniaceae. P. graveolens es un arbusto aromático perenne de alto valor que puede alcanzar una altura de hasta 1,3 metros. El aceite esencial de $P$. graveolens es ampliamente utilizado en la perfumería y cosmética (Misra y Srivastava, 2010). Diversos estudios han demostrado que el aceite de geranio contiene como constituyentes mayoritarios el citronelol $(19,3-40,2 \%)$ y geraniol $(6,5-18,4 \%)$ (Gomes et al., 2007). El aceite esencial de citronela es utilizado ampliamente en la industria cosmética, principalmente en perfumes y en la industria alimentaria como saborizante y conservante (Atailia y Djahoudi, 2015). Algunas investigaciones han reportado su actividad antiinflamatoria, antioxidante, hipoglucemiante, antimicrobiana e inmunoestimulante (Boukhris et al., 2012; Boukhatem et al., 2013).

Para la producción de nuevos individuos de plantas se utiliza la micropropagación, que puede ser sexual o asexual. Esta última es la multiplicación de una planta a partir de una célula, un tejido o un órgano, produciendo individuos a partir de explantes.
Se obtienen clones de la planta, conservando las características del individuo, es decir, de la planta madre (Garay y Echeverría, 2010). La ventaja de esta técnica es que se produce un gran número de ejemplares a partir de una sola planta en espacios reducidos (Lemes-Hernández et al., 2001). La propagación por estacas, es muy frecuente en especies de importancia forestal, florística, agrícola y agroindustrial (Hernández et al., 2005; Herrera et al., 2007; Ruiz y Mesén 2010; Ordóñez et al., 2012). El objetivo de este trabajo fue estudiar la propagación de explantes (hojas) de citronela (Pelargonium graveolens) y su establecimiento bajo malla sombra.

\section{Materiales y métodos}

\section{Ubicación del área de estudio}

Este trabajo se llevó a cabo durante el ciclo primavera-verano del 2017, en el vivero del Departamento de Investigaciones Científicas y Tecnológicas de la Universidad de Sonora, ubicado en las coordenadas $29^{\circ} 05^{\prime} 00^{\prime \prime}$ latitud $\mathrm{N} 110^{\circ}$ 57'51' longitud oeste.

\section{Área seleccionada}

Se acondicionó un área de $40 \mathrm{~m}^{2}$, cubierta con malla sombra de $80 \%$ de luz con un tipo de suelo arcilloso y escasa materia orgánica.

\section{Material vegetal}

Los explantes (hojas) se colectaron de plantas adultas de citronela (Pelargonium graveolens) ya existentes en el área de estudio con una edad promedio de cuatro meses. Para ello se utilizaron unas tijeras para podar, las cuales se desinfectaron previamente con cloro al $10 \%$.

\section{Labores agronómicas}

Con la finalidad de airear el suelo se removió la tierra y se levantaron surcos para el previo establecimiento de las nuevas plántulas de citronela.

\section{Instalación del sistema de riego}

Considerando las altas temperaturas que se registran en Hermosillo, Sonora, México y las bajas precipitaciones al año se instaló un sistema 
de riego por goteo, con el fin de generar un bulbo de humedad en la parte radicular de las plántulas. El gasto por planta fue de $1 \mathrm{lph}$ por gotero. Se aplicó media hora de riego cada dos días.

\section{Densidad de plantas}

Se manejó una densidad de 42 plantas. La distancia entre cada planta fue de $50 \mathrm{~cm}$ y 1 metro entre cada surco.

\section{Tratamiento}

Para evaluar el comportamiento de acuerdo con el tiempo de enraizamiento se utilizaron dos tratamientos como sustrato (suelo y pea moss).

\section{Trasplante de los explantes (hojas) en suelo y macetas}

Los explantes (hojas) que se utilizaron para este estudio medían de 18 a $23 \mathrm{~cm}$ de longitud (considerando el peciolo). Se sembraron previamente en el suelo y en charolas con peat moss.

\section{Parámetros a evaluar}

Porcentaje de enraizamiento. El porcentaje de enraizamiento se evaluó en los explantes (hojas) de citronela sembrados en el suelo y peat moss de acuerdo con Garay y Echeverría (2013).

Porcentaje de sobrevivencia al trasplante. Se evaluó en explantes (hojas) sembradas en el suelo y peat moss de acuerdo con Mc Caughey-Espinoza et al. (2018).

Longitud de raíz principal. Para eliminar el sustrato (suelo o peat moss) las raíces fueron previamente lavadas y se midió la longitud de la raíz de citronela desde la base del tallo hasta el ápice de la raíz más larga, utilizando una cinta métrica graduada en cm (Garay y Echeverría, 2013).

Número de raíces secundarias. Únicamente se consideraron las raíces primarias sin tener en cuenta las secundarias y terciarias (Garay y Echeverría, 2013).

Mediciones dasométricas. Por ser un tipo de planta aromática y medicinal se consideró evaluar el comportamiento respecto a su altura, cobertura aérea y tallo (Mc Caughey et al., 2017). Las mediciones se llevaron a cabo mediante la toma de datos de 10 plantas al azar de cada tratamiento. La medición del diámetro basal se realizó al ras del suelo y el diámetro de copa se obtuvo al medir el diámetro de copa. Para ello se utilizó una cinta métrica graduada en $\mathrm{cm}$ y basal de las plantas, y se expresaron en $\mathrm{cm}^{2}$ o cm. Se aplicó la siguiente ecuación:

$$
\begin{array}{ll}
\mathrm{a}=\mathrm{Xd}^{*} \pi / 2 \text { Donde: } & \mathrm{a}=\text { Área } \\
& \mathrm{Xd}=\text { Diámetro } \\
& \pi=3.1416
\end{array}
$$

Rendimiento de materia seca (RMS). Se llevó a cabo de acuerdo a Luna-Guerrero et al. (2018). Se tomó el peso de 10 plantas ya establecidas al azar de cada uno de los tratamientos. Se calculó de acuerdo al peso seco por planta (g).

Plagas y enfermedades presentes en las plantas. Se monitoreó durante 6 meses, que fue el tiempo en el cual se realizó este estudio.

\section{Diseño experimental}

Para este estudio se utilizó un diseño completamente aleatorizado. La variable a evaluar fue el porcentaje de enraizamiento de los explantes (hojas) en suelo y peat moss. Cada tratamiento presentó cuatro repeticiones y en cada una de ellas se evaluaron 25 esquejes. Por lo tanto, se observaron 100 explantes por tratamiento. Se realizó un análisis de varianza (ANDEVA) con un nivel de significancia de $\mathrm{p}<0,05$ y una comparación de medias con la prueba de Tukey-Kramer con un alfa de $0,05 \%$. Se hizo un análisis de correlaciones de Pearson. Estos análisis se llevaron a cabo con el paquete estadístico JMP 11 (JMP, 2011).

\section{Resultados y discusión}

\section{Porcentaje de enraizamiento de los explantes (hojas)}

El porcentaje de enraizamiento de las hojas de citronela en el suelo y peat moss presentó diferencias significativas $(\mathrm{p}<0.05)$. Las hojas sembradas en el suelo mostraron un mayor enraizamiento (91\%) comparado con las hojas que se colocaron en macetas con peat moss como sustrato (33\%) (Figura 1). La mayoría de los explantes (hojas) enraizados en suelo produjeron brotes. Sin embargo, el bajo porcentaje de enraizamiento en el sustrato de peat moss se puede atribuir a la falta de drenaje, lo que ocasionó pudrición en el peciolo de las hojas. Esto 


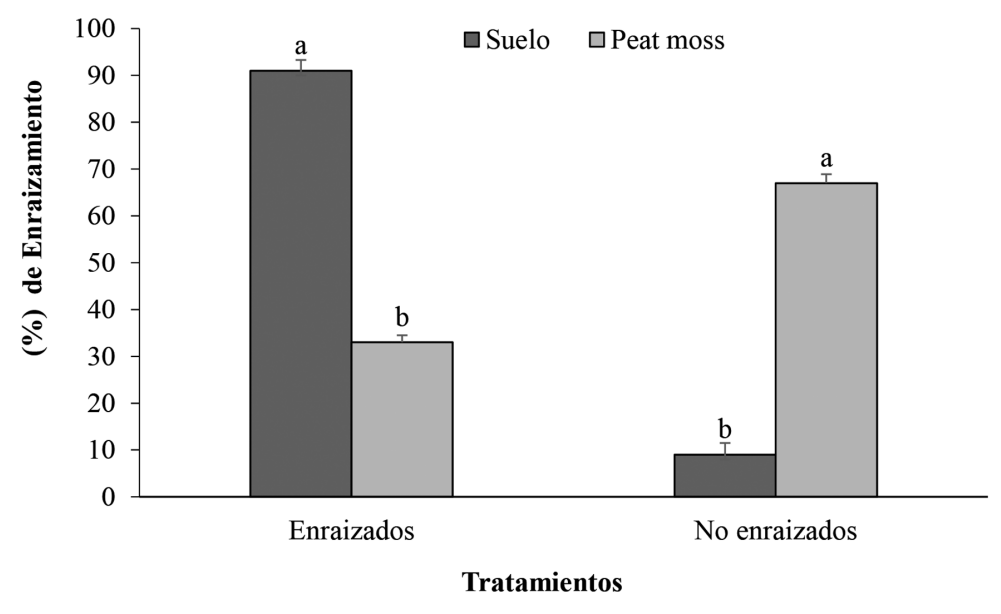

Figura 1. Porcentaje de enraizamiento de explantes (hojas) de citronela crecidas en suelo y peat moss. Diferentes letras entre tratamientos indican diferencias significativas $(\mathrm{p}<0,05)$.

se debe a que el peciolo de las hojas de citronela no es lignificado y es hueco en su interior. Además, el sustrato de peat moss no contiene nutrientes.

El alto porcentaje de enraizamiento de las hojas de citronela en el suelo se atribuye a cierta cantidad de nutrientes que generan raíces verdaderas no artificiales (poco lignificadas de color blanco). Además, en el peciolo de las hojas se producen hormonas como las auxinas, que son responsables de inducir el proceso de desarrollo de raíces (Herrera et al., 2007). El enraizamiento presenta una tendencia de un movimiento polar de auxinas que se transportan con más facilidad a la parte inferior (Bandurski et al., 1992; LemesHernández et al., 2001). La formación de raíces en los nuevos individuos también se favorece al reducir la transpiración y, por ende, mejora los procesos de fotosíntesis, tipo de sustratos y fitohormonas (Ruiz y Mesén, 2010). En este estudio se utilizó la hoja completa de Pelargonium graveolens, la cual mostró un alto porcentaje de enraizamiento (arriba del 90\%), al proporcionar auxinas, vitaminas, carbohidratos, nitrógeno orgánico y realizando la fotosíntesis (Herrera et al., 2007; Bandurski et al., 1992; Lemes-Hernández et al., 2001).

En la propagación de las especies vegetales es importante considerar la edad del explante. Garay y Echeverría (2013) recomiendan utilizar partes vegetales que provengan de plantas jóvenes. Igualmente, Kochhar et al. (2008) mencionan que la época de recolección, la composición bioquímica y la condición fisiológica de la planta madre influyen en la respuesta al enraizamiento. Algunos estudios han mostrado la formación de raíces en diferentes lugares de la misma rama (Kochhar et al., 2008).

Existen diversas investigaciones en las cuales se ha logrado enraizar diferentes plantas aplicando reguladores de crecimiento. Solís et al. (2015) reportaron un $92 \%$ de enraizamiento en estacas de $6 \mathrm{~cm}$ de longitud de azul de mata (Justicia tinctoria) sembradas bajo un ambiente protegido, utilizando ácido indolbutírico en concentraciones de 1000 y 1500 ppm con diferentes sustratos. Ruiz y Mesén (2010) obtuvieron $80 \%$ de enraizamiento en esquejes de Sacha Inchi (Plukenetia volubilis L.) crecidoss con distintas concentraciones de ácido indolbutírico.

Ordóñez et al. (2012) propagaron esquejes de lulo silvestre (Solanum hirtum Vahl, S. marginatum L. f., S. sessiliflorum Dun, S. mammosum L. y S. umbellatum Mill) utilizando ácido naftalenacético y obtuvieron un $62,5 \%$ de enraizamiento en $S$. sessiliflorum y $61,9 \%$ en $S$. marginatum. Sin embargo, en este estudio no se utilizaron reguladores de crecimiento vegetal sintético de ningún tipo (auxínico, citocínico y giberelínico). Por lo tanto, la citronela (Pelargonium graveolens) es una planta viable para su propagación utilizando las hojas completas para la obtención de nuevos ejemplares. En consecuencia, es importante considerar la época de propagación de esta planta.

\section{Sobrevivencia al trasplante}

De manera general, las plántulas de citronela (Pelargonium graveolens) producidas tanto en suelo como en peat moss presentaron un $91 \%$ de sobrevivencia al trasplante, de acuerdo a las 
condiciones climatológicas existentes (temperaturas promedio de $33{ }^{\circ} \mathrm{C}$ ). Por ello podemos considerar aceptable este porcentaje de sobrevivencia al trasplante. En la Figura 2 A-B se observa el establecimiento y climatización de citronela en el área de estudio.

Al obtener un buen porcentaje de sobrevivencia al trasplante, esta especie demuestra que es de fácil adaptación a las temperaturas presentes en el estado de Sonora, México. La humedad constante que se aplicó de acuerdo a los tres riegos para asegurar el trasplante fue importante para generar un microclima, así como las labores agronómicas (Mc Caughey-Espinoza et al., 2017).

\section{Longitud de raíz principal y número de raíces secundarias}

No se presentaron diferencias significativas ( $p>0,05)$ con respecto a la longitud de raíz principal y el número de raíces secundarias en explantes de hoja de citronela (Pelargonium graveolens) al utilizar suelo o peat moss. Se observó una correlación positiva $(\mathrm{r}=0,88)$ entre la longitud de las raíces y el número de raíces secundarias (Figura 3). La raíz es la parte más importante de la planta, por lo tanto deberá soportar el peso de la biomasa verde o seca aérea (Mc Caughey-Espinoza et al., 2018). Además, los métodos de aplicación de riegos pueden afectar el desarrollo de las raíces. Por lo tanto, los factores no deseables impactarán directa o indirectamente en el crecimiento, desarrollo y calidad del sistema radicular de las plantas y, por ende, en su potencial de producción (Davies y Zhang, 1991; Glenn, 2000). Generalmente los esquejes más grandes presentan raíces adventicias con mayor longitud, al contener mayor reservas para la inducción de raíces (Hernández et al., 2005). El desarrollo radicular se ve afectado por

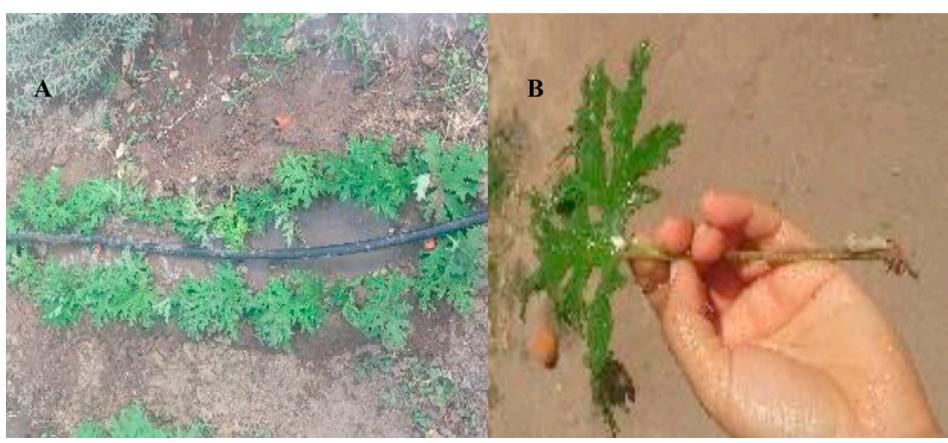

Figura 2. A) Proceso de enraizamiento de citronela. B) Plántula de citronela.

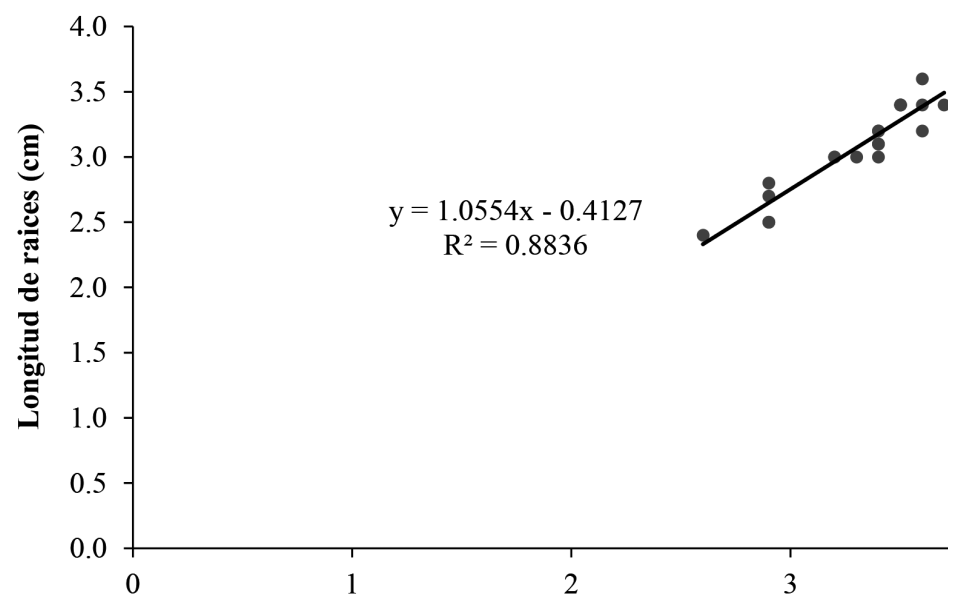

Figura 3. Correlación de número de raíces secundarias y longitud de raíces en hojas de citronela (Pelargonium graveolens). 
factores como humedad, aireación, temperatura y resistencia mecánica en los suelos (Callejas et al., 2010).

De acuerdo con Fachinello et al. (2005), es recomendable utilizar estacas lignificadas porque la lignificación está asociada a la reserva de carbohidratos. Por otra parte, Hartmann y Kester (2010) reportaron que la inducción de raíces fue mejor en estacas apicales por la concentración de sustancias endógenas que son promotoras del enraizamiento. Similarmente, en este trabajo se observó que en el peciolo de las hojas de citronela (Pelargonium graveolens) existe una cantidad considerable de estas sustancias para la generación de raíces, por lo tanto, los resultados no concuerdan con Fachinello et al. (2005).

Con los cambios evolutivos que se están originando por el calentamiento global, la agricultura demanda generar otras vías de producción sostenible que beneficien al productor, a la sociedad y al medio ambiente, así como a la industria agromercantil que requiere un mayor cultivo de plantas aromáticas y medicinales. En la actualidad existe nula información sobre la propagación y establecimiento de citronela (Pelargonium graveolens), y de acuerdo a los resultados obtenidos, esta especie pudiera ser un cultivo prometedor para el estado de Sonora, México.

\section{Medidas dasométricas y rendimiento}

Las plántulas de citronela (Pelargonium graveolens) producidas en ambos sustratos (peat moss y suelo) no presentaron diferencias significativas $(p>0,05)$ en las mediciones dasométricas (altura, diámetro de copa y basal). Las plantas alcanzaron una altura de 44,3 a 46,89 cm, un diámetro de copa de 31,3 a $33,2 \mathrm{~cm}^{2}$ y diámetro basal de 1,20 a $1,33 \mathrm{~cm}^{2}$ (Tabla 1). También mostraron un desarrollo y crecimiento aceptables. De acuerdo a los resultados obtenidos, se puede estimar una densidad de siembra de citronela de 16,667 plantas $\mathrm{ha}^{-1}$, y posibles rendimientos de 1,856.6 a 1,075.5 kg/ha-1. En la Figura 4 se observan las plantas de citronela que se cosecharon en el suelo y peat moss.

Se encontraron diferencias significativas $(\mathrm{p}<0,05)$ en los rendimientos de materia seca de plantas de citronela de $34.6 \mathrm{~g}$ (plantas enraizadas en el suelo), mientras que las plantas que se enraizaron en peat moss y posteriormente se trasplantaron al suelo obtuvieron un promedio de $32,1 \mathrm{~g}$. Este comportamiento puede atribuirse a que las plantas enraizadas en peat moss tuvieron que

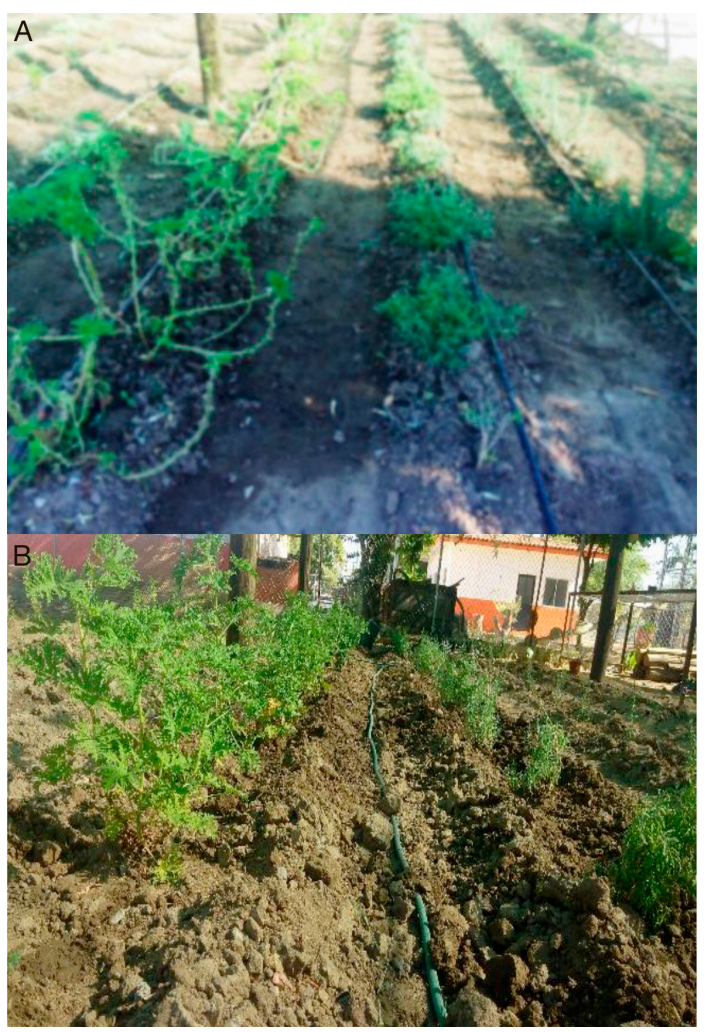

Figura 4. Cosecha de citronela (Pelargonium graveolens) A) Peat moss y B) Suelo.

Tabla 1. Medidas dasométricas y rendimiento de materia seca de citronela (Pelargonium graveolens) ${ }^{\mathrm{a}}$

\begin{tabular}{lcccc}
\hline & \multicolumn{2}{c}{ Mediciones dasométricas } & Rendimiento \\
\hline Sustratos & Altura $(\mathrm{cm})$ & Diámetro de copa $\left(\mathrm{cm}^{2}\right)$ & Diámetro basal $\left(\mathrm{cm}^{2}\right)$ & Materia seca $(\mathrm{g})$ \\
Suelo & $46,2 \pm 0,539^{\mathrm{A}}$ & $32,6 \pm 0,653^{\mathrm{A}}$ & $1,3 \pm 0,014^{\mathrm{A}}$ & $34,6 \pm 0,847^{\mathrm{A}}$ \\
Peat moss & $46,1 \pm 0,732^{\mathrm{A}}$ & $32,1 \pm 0,740^{\mathrm{A}}$ & $1,2 \pm 0,045^{\mathrm{A}}$ & $32,1 \pm 0,450^{\mathrm{B}}$ \\
\hline
\end{tabular}

${ }^{a}$ Diferentes letras mayúsculas entre tratamientos indican diferencias significativas $(p<0,05)$. 
adaptarse a otro tipo de sustrato (suelo), mientras que las plántulas que fueron enraizadas en suelo no presentaron este problema (Tabla 1).

\section{Plagas y enfermedades}

Durante las etapas de climatización y establecimiento de los explantes (hojas) de citronela (Pelargonium graveolens), que se extendieron por 6 meses, se realizó un monitoreo de la presencia de plagas y enfermedades. No se detectaron plagas ni enfermedades en los explantes de hojas en ambos sustratos. A la planta de citronela se le han atribuido efectos repelentes naturales contra insectos. Además se debe considerar que fue la primera plantación de citronela en esta área de estudio.

\section{Conclusiones}

Los explantes (hojas) de citronela (Pelargonium graveolens) enraizados en suelo presentaron raíces más firmes que las obtenidas en peat moss. Además no fue necesario el uso de fitohormonas para el enraizamiento en hojas de citronela. Durante el proceso de climatización y establecimiento de los explantes no se reportaron plagas ni enfermedades en suelo y peat moss. La propagación vegetativa de hojas de citronela fue posible y permitió la obtención de nuevas plantas homogéneas de manera fácil y a bajo costo. Sin embargo, la propagación se vio favorecida en las hojas sembradas en suelo, lo cual podría beneficiar a los productores de hierbas aromáticas en el estado de Sonora.

\section{Literatura citada}

Atailia, I.; Djahoudi, A.

2015. Composition chimique et activité antibactérienne de l'huile essentielle de géranium rosat (Pelargonium graveolens L'Hér.) cultivé en Algérie. Phytothérapie, 13(3): 156-162.

Bandurski, R.S.; Schulze, A.; Jensen, P.; Desrosiers, M.; Epel,

B.; Kowalczyk, S.

1992. The mechanism by which an asymmetric distribution of plant growth hormone is attained. Adv. Space Res, 12(1): 203-210

Boukhatem, M.; Kameli, A.; Ferhat, M.A.; Saidi, F.; Mekarnia, M. 2013. Rose geranium essential oil as a source of new and safe anti-inflammatory drugs. Libyan J. Med. 8: 22520-22526.

Boukhris, M.; Bouaziz, M.; Feki, I.; Jemai, H.; El Feki, A.;

Sayadi, S.

2012. Hypoglycemic and antioxidant effects of leaf essential oil of Pelargonium graveolens L'Hér. In alloxan induced diabetic rats. Lipids Health Dis, 11: 81-90.

Callejas, R.R.; Canales, P.P.; de Cortázar, V.G.G.D.

2010. Relationship between root growth of "Thompson Seedless" grapevines and soil temperature. Chilean J. Agric. Res, 69(4): 496-502.

Davies, W.J.; Zhang, J.

1991. Root signals and the regulation of growth and development of plants in drying soil. Annual Review of Plant Physiology and Plant Molecular Biology, 42(1): 55-76.

Fachinello, J.C.; Hoffman, A.; Nachtigal, J.

2005. Propagação vegetativa por estaquia. En: Fachinello, JC. Propagação de Plantas Frutíferas. Brasilia, DF. Embrapa Informação Tecnológica (Eds). 69-109 p.

Garay, C.R.E.; Echeverría, F.M.C.

2013. Propagación vegetativa de Jatropha curcas L. por estacas. Investigación Agraria, 12(2): 69-73.

Glenn, D.M.

2000. Physiological effects of incomplete rootzone wetting on plant growth and their implications for irrigations management. HortScience, 35(6):1041-1043.

Gomes, P.B.; Mata, V.G.; Rodrigues, A.E.

2007. Production of rose geranium oil using supercritical fluid extraction. The Journal of Supercritical Fluids, 41(1): 50-60.
Hartmann, H.D.; Kester, F.D.; Davies, F.T.; Geneve, R.L. 2010. Hartmann and Kester's plant propagation. Principles and practices. Pearson Education (Eds). 760 p.

Hernández, J.R.; Tatis, H.A.; Ayala, C.E.C.

2005. Influencia del ácido indolbutírico y ácido naftalenoacético sobre el enraizamiento de esquejes de caña flecha (Gynerium sagittatumaubl.). Temas agrarios, 10(1): 5-13.

Herrera, J.G.Á.; Rodríguez, S.L.; Chacón, E.

2007. Efecto de diferentes tamaños de esqueje y sustratos en la propagación del romero (Rosmarinus officinalis L.). Agronomía Colombiana, 25(2): 224-230.

Juárez, C.; Aguilar, J.; Juárez, M., Bugarin, R.; Juárez, L.P.; Cruz, E.

2013. Hierbas aromáticas y medicinales en México: Tradición e Innovación. Bio Ciencias, 2(3): 119-129.

JMP.

2011. The Statistical Discovery Software. SAS Institute Inc. Ver. 11.

Kochhar, S.; Singh, S.P.; Kochhar, V.K.

2008. Effect of auxins and associated biochemical changes during clonal propagation of the biofuel plant Jatropha curcas. Biomass and Bioenergy, 32(12): 1136-1143.

Lemes-Hernández, C.M.; Rodríguez-Ferradá, C.A.; Acosta de la Luz, L.

2001. Multiplicación vegetativa de Rosmarinus officinalis L. (romero). Rev. Cubana Plant Med, 6(3):79-82.

Luna-Guerrero, M.J.; López-Castañeda, C.; Hernández-Garay, A.; Martínez-Hernández, P.A.; Ortega-Cerrilla, M.E.

2018. Evaluación del rendimiento de materia seca y sus componentes en germoplasma de alfalfa (Medicago sativa L.). Revista Mexicana de Ciencias Pecuarias, 9(3): 486-505.

Mc Caughey-Espinoza, D.M.; Ayala Astorga, G.I.; VelázquezCaudillo, J.; Anaya-Islas, J.; Canseco-Vilchis, E.

2017. Creación de un jardín botánico y de árbol madre de arbustivas forrajeras nativas del estado de Sonora. Idesia (Arica), 35(4), 35-45. 
Mc Caughey-Espinoza, D.M.; Ayala-Astorga, G.I.; BurboaZazueta, M.G.; Retes-López, R.; Ochoa-Meza, A.

2018. Uso de plantas nativas para la rehabilitación de canteras en Sonora. Idesia (Arica), 36(4): 17-24.

Misra, A.; Srivastava, N.K.

2010. Valor agregado de esencial aceite(s) de monoterpeno en geranio (Pelargonium graveolens) en posiciones de hoja para explotación comercial. Revista Africana de Investigación Agrícola, 5(15): 2077-2079.

Ordóñez, U.C.; Gómez, O.H.; Ordóñez, J.H.R.; Lagos, B.T.C. 2012. Evaluación de un sistema de propagación vegetativa mediante esquejes en lulo silvestre Solanum hirtum Vahl, S. marginatum L.f., S. sessiliflorum Dun, S. mammosum L. y S. umbellatum Mill. Revista de Ciencias Agrícolas, 29(1): 29-41.
Pérez, C.R.

2009. Frutas y hortalizas orgánicas de la red de mercados y tianguis orgánicos de México. Estudio del Sial. Claridades Agropecuarias, 194: 25-45.

Ruiz-Solsol, H.; Mesén, F.

2010. Efecto del ácido indolbutírico y tipo de estaquilla en el enraizamiento de sacha inchi (Plukenetia volubilis L.). Agronomía Costarricense, 34(2): 269-285.

Shankar, P.R.; Balasubramanium, R.

2014. Antimicrobial resistance: global report on surveillance 2014. Australasian Medical Journal, 7(5): 237.

Solís, C.A.; Jiménez, V.; Arias, J.

2015. Propagación asexual de azul de mata (Justicia tinctoria (Oerst.) DN Gibson, Fam. Acanthaceae) por medio de estacas. Agronomía Costarricense, 39(2): 91-104. 